\title{
ESCLERODERMIA SISTÊMICA E DOENÇAS ASSOCIADAS: UM RELATO DE CASO
}

Systemic Sclerodermia and Associated Diseases: a case report

Esclerodermia Sistémica y Enfermedades Asociadas: informe de un caso

\section{Caio Willer Brito Gonçalves*1, Dilomá Bastos Alves Júnior², Daniel}

Laureano de Castro ${ }^{3}$, Rafael Felipe Carvalho Canuto ${ }^{4}$, Janne Marques Silveira ${ }^{5}$, Andreia de Kássia Lemos de Brito ${ }^{6}$.

${ }^{1,2,3}$ Graduando em Medicina, Universidade de Gurupi (UNIRG), Gurupi, Tocantins, Brasil.

${ }^{4}$ Médico, Universidade Federal do Tocantins (UFT), Palmas, Tocantins, Brasil.

${ }^{5}$ Fisioterapeuta, Mestre em reabilitação, Especialista em fisiologia cardiovascular e respiratória, Docente do curso de medicina e fisioterapia, Universidade de Gurupi (UNIRG), Gurupi, Tocantins, Brasil.

${ }^{6}$ Enfermeira, Mestranda em ciências da saúde, Especialista em gestão em saúde, Docente do curso de enfermagem, Universidade de Gurupi (UNIRG), Gurupi, Tocantins, Brasil.

*Correspondência: Universidade de Gurupi, Av. Rio de Janeiro, $N^{\circ} 1585$ - St. Central, Gurupi, Tocantins, Brasil. CEP:77.403-090.e-mail Caiowillerb@gmail.com

Artigo recebido em 29/10/2019 aprovado em 10/02/2020 publicado em 24/03/2020.

\section{RESUMO}

Esclerodermia Sistêmica (ES) é uma patologia rara, autoimune com evolução multissistêmica. Relata-se o caso de uma paciente, L. S. R., negra, 41 anos de idade, residente em Gurupi-TO que compareceu à Unidade Básica de Saúde (UBS) do bairro Vila Nova no município de Gurupi-TO. Durante a anamnese, paciente relatou ser portadora de ES associada a complicações como Pneumopatia Intersticial, Artrite Reumatoide e Doença do Refluxo Gastresofágico. O prontuário indicou que o diagnóstico foi feito no dia 6 de agosto de 2018, baseado na presença das manifestações clínicas de úlceras digitais, esclerodactilia, fenômeno de Raynaud, microstomia e leucomelanodermia. Aos exames de imagem, a tomografia computadorizada evidenciou padrão de vidro fosco bilateral compatível com Pneumopatia Intersticial. Esofagograma com dilatação esofagianas e preenchimento contínuo e fase tardia com retardo do esvaziamento por redução do calibre, compatível com Doença do Refluxo Gastresofágico. Foi feita uma intervenção terapêutica com razoável melhora do quadro da paciente, através de medicações como Ciclofosfamida, Nifedipino Retard, Captopri, Omeprazol, Domperidona, Carbonato de Cálcio + Vitamina D. Mesmo identificando melhora dos sintomas relatados pela paciente na consulta do dia 20 de setembro de 2019, faz se necessários maiores estudos sobre a patogênese dessa afecção, bem como explorar as medidas terapêuticas eficazes.

Palavras-chave: Escleroderma Sistêmico. Pneumopatias. Evolução Clínica.

\section{ABSTRACT}

Systemic scleroderma (SSc) is a rare, autoimmune pathology with multisystemic evolution. We report the case of a 41-year-old black woman, LSR, resident in Gurupi-TO who attended the Basic Health Unit (UBS) of the Vila neighborhood. New in the municipality of Gurupi-TO. During the anamnesis, a patient reported having SSc associated with complications such as interstitial lung disease, rheumatoid arthritis and gastroesophageal reflux disease. The medical record indicated that the diagnosis was made on August 6, 2018, based on the presence of the clinical manifestations of digital ulcers, sclerodactyly, Raynaud's phenomenon, microstomy and leukomelanodermia. At the imaging exams, the computed tomography showed a compatible bilateral ground-glass pattern. with interstitial lung disease. Esophagogram with esophageal dilatation and continuous filling and late phase with delayed emptying due to reduction in size, compatible with Gastroesophageal Reflux Disease. Therapeutic 
intervention was performed with reasonable improvement of the patient's condition through medications such as Cyclophosphamide, Nifedipino Retard, Captopri, Omeprazole, Domperidone, Calcium Carbonate + Vitamin D. Even identifying improvement of symptoms reported by the patient at the September 20th consultation. In 2019, further studies on the pathogenesis of this condition are needed, as well as to explore effective therapeutic measures. Keywords: Systemic scleroderma. Lung diseases. Clinical evolution.

\section{RESUMEN}

La esclerodermia sistémica (SSc) es una patología autoinmune rara con evolución multisistémica. Presentamos el caso de una mujer negra de 41 años, LSR, residente en Gurupi-TO que asistió a la Unidad de Salud Básica (UBS) del barrio de Vila. Nuevo en el municipio de Gurupi-TO. Durante la anamnesis, un paciente informó que tenía SSc asociado con complicaciones tales como enfermedad pulmonar intersticial, artritis reumatoide y enfermedad por reflujo gastroesofágico. El registro médico indicó que el diagnóstico se realizó el 6 de agosto de 2018, con base en la presencia de manifestaciones clínicas de úlceras digitales, esclerodactilia, fenómeno de Raynaud, microstomía y leucomelanodermia.En los exámenes de imágenes, la tomografía computarizada mostró un patrón bilateral compatible de vidrio esmerilado. con enfermedad pulmonar intersticial. Esofagograma con dilatación esofágica y llenado continuo y fase tardía con vaciado tardío debido a la reducción de tamaño, compatible con la enfermedad por reflujo gastroesofágico. La intervención terapéutica se realizó con una mejora razonable de la condición del paciente a través de medicamentos como ciclofosfamida, nifedipino retard, captopri, omeprazol, domperidona, carbonato de calcio + vitamina D. Incluso identificando una mejoría de los síntomas informados por el paciente en la consulta del 20 de septiembre. En 2019, se necesitan más estudios sobre la patogénesis de esta afección, así como para explorar medidas terapéuticas efectivas.

Descriptores: Esclerodermia sistémica. Enfermedades pulmonares. Evolución clínica

\section{INTRODUÇÃO}

Esclerodermia Sistêmica (ES) é uma patologia relacionada à autoimunidade e com evolução para o acometimento multissistêmico (AZULAY, 2017). É caracterizada por modificações da microcirculação podendo causar fibrose e obliteração das veias e artérias da pele, pulmão, trato gastrintestinal e rins. Entre as complicações que podem elevar a morbimortalidade da doença, encontra-se a crise renal esclerodérmica, hipertensão pulmonar e a pneumopatia intersticial (JACQUIER et al., 2019).

Este estudo visa relatar o caso de uma paciente diagnosticada com ES associada à Pneumopatia Intersticial (PI), Artrite Reumatoide (AR), Doença do Refluxo Gastresofágico (DRGE). Os dados foram coletados através de registros médicos apresentados pela paciente, revisão de prontuário, anamnese e exame físico. Os achados clínicos foram registrados por fotografia, devidamente autorizados pela paciente, onde a mesma assinou um termo de consentimento livre e esclarecido (TCLE) que foi aprovado pelo
Comitê de Ética em Pesquisa da Universidade de Gurupi com o parecer 3.666.275 e o CAAE 23724619.0.0000.5518.

\section{RELATO DO CASO}

L. S. R., sexo feminino, negra, 41 anos, $74 \mathrm{Kg}$, PA de $150 \times 70 \mathrm{mmHg}$ em repouso e altura de 1,49 metro, residente em Gurupi-TO. Compareceu à Unidade Básica de Saúde (UBS) do bairro Vila Nova no município de Gurupi-TO, apresentando queixa principal de dor em membros superiores e membros inferiores. Durante a anamnese relatou ter sido diagnosticada há cerca de 1 ano com ES e as seguintes complicações: PI, DRGE, AR.

Durante a consulta na UBS foi realizada revisão do prontuário da paciente e extraído os dados que embasaram o diagnóstico da doença. Consoante prontuário, foi internada no Hospital Regional de Gurupi (HRG) no dia 02 de agosto de 2018 com dor intensa ( 9 pontos na escala numérica de dor) e edema 
em falanges distais de ambos os pés e $2^{\circ}$ pododáctilo do pé direito.

No dia 4 de agosto foi encaminhada para avaliação especializada em reumatologia no Hospital Geral de Palmas (HGP), onde foi identificado presença de AR nas articulações interfalangianas proximais (IFP) e em joelhos bilateralmente, além da presença de úlceras digitais e estiramento da pele. Pelo fato da paciente haver histórico prévio de hanseníase, suspeitou-se de quadro de recidiva sendo iniciado o tratamento com corticoide.

Recebeu alta do HGP em 05 de agosto, sendo encaminhada para o ambulatório de dermatologia da Universidade de Gurupi para acompanhamento do tratamento de suposta recidiva da hanseníase. Compareceu ao ambulatório no dia 06 de agosto, no qual foi avaliada pela dermatologista e grupo de internos que afastou a hipótese de recidiva de hanseníase e fez o diagnóstico de ES, baseado nas manifestações clínicas de úlceras digitais, esclerodactilia, fenômeno de Raynaud, microstomia e leucomelanodermia.

A paciente foi encaminhada para o HGP novamente, onde no dia 7 de agosto, adotou-se uma intervenção terapêutica para PI através da prescrição da infusão da Ciclofosfamida $1 \mathrm{~g}$, sem intercorrências.

No dia 8 de agosto de 2018 a paciente teve alta hospitalar do HGP, sendo prescrito para uso em casa de Nifedipino Retard $20 \mathrm{mg}$ de $8 \mathrm{em} 8$ horas, Captopril $25 \mathrm{mg}$ de 12 em 12 horas, Omeprazol 40 mg 12 em 12 horas, Domperidona $10 \mathrm{mg} 3$ vezes ao dia e Carbonato de Cálcio associado à Vitamina D 1 comprimido após o almoço, além de orientações quanto ao desmame da Prednisona.

No momento da alta hospitalar, apresentava infecção na pele de dois pododáctilos do pé direito sendo também prescrito para uso em casa de Cefalexina $500 \mathrm{mg}$ de 6 em 6 horas por 5 dias.
Foi orientada a fazer para avaliações periódicas em UBS do município de Gurupi e orientada a realizar coleta de sangue para hemograma entre os dias 17 a 21 de agosto de 2018 para avaliação da dose da próxima Ciclofasfamida prevista para o dia 6 de setembro de 2018.

$\mathrm{Na}$ consulta da UBS realizada em 20 de setembro de 2019, paciente relatou irritabilidade, queda de cabelo, hiperemia ocular pela manhã, melhora significativa das úlceras digitais e redução das manifestações dos FRy.

Com relação aos aspectos gastrointestinais, refere sensação de refluxo, epigastralgia e constipação intestinal há dois anos. Em relação aos tecidos osteomuscular refere dor em articulações IFP e apresenta edema de membros inferiores.

Foi interrogada sobre os antecedentes ginecológicos e obstétricos sendo relatado amenorreia há aproximadamente um ano, associada de irritabilidade, fogachos esporádicos e duas gestações com partos normais, sem abortos. Aos antecedentes patológicos refere diagnóstico de hipertensão arterial há 15 anos em uso atualmente de Captopril 25 mg de 12/12 horas e que teve diagnóstico de hanseníase no ano de 2007, sendo feito o esquema terapêutico ao longo daquele ano. Nega etilismo e tabagismo.

Ao exame físico apresentou-se em bom estado geral, lúcida e orientada em tempo e espaço, eupneica, afebril, com presença de afinamento do nariz e microstomia. No exame cardiovascular apresentou ritmo cardíaco regular, em dois tempos com hiperfonese de segunda bulha cardíaca, sem sopro.

Ao exame respiratório apresentou murmúrio vesicular, sem ruídos adventícios. No exame osteoarticular houve presença de edema e sinais flogísticos em falanges distais e $2^{\circ}$ pododáctilo do pé direito.

Ao exame nos membros apresentou de esclerodactilia em membros, leucomelanodermia em 
membros superiores (Figuras 1 e 2), região tibial anterior (Figura 3), região distal de perna direita e esquerda (Figura 3) e face medial da coxa esquerda (Figura 4). Apresentou pulso pedioso e tibial posterior palpáveis e panturrilhas livres.

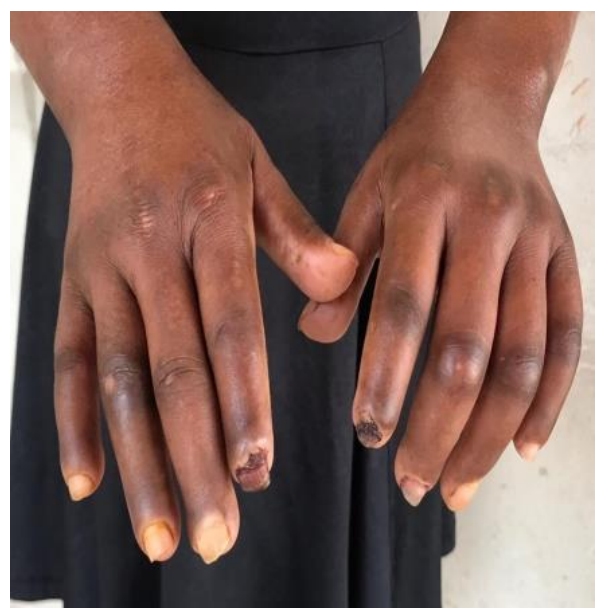

Figura 1. Presença de esclerodactilia, Leucomelanodermia, endurecimento e espessamento da pele no dia 20 de setembro de 2019.

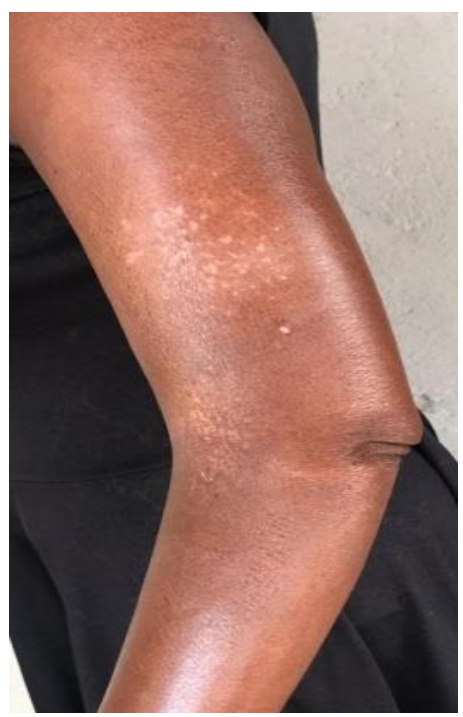

Figura 2. Presença de Leucomelanodermia, espessamento e enrijecimento da pele de membro superior.

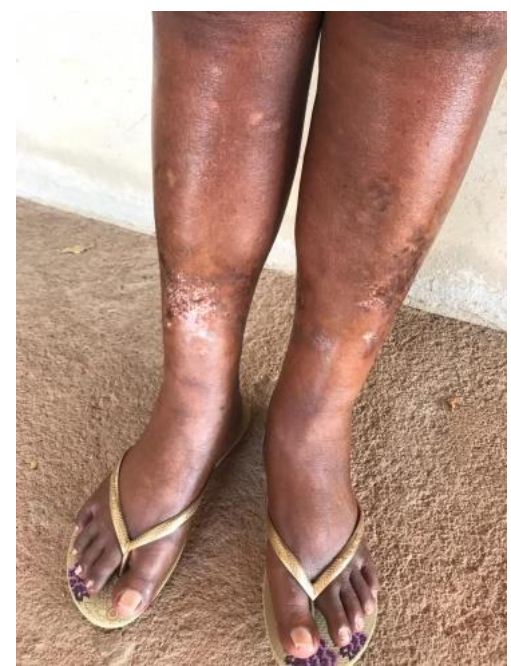

Figura 3. Presença de Leucomelanodermia, espessamento e enrijecimento da pele de membros inferiores.

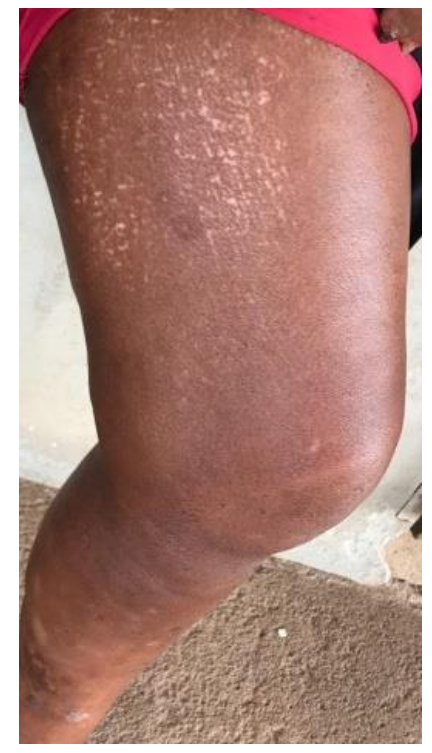

Figura 4. Presença de Leucomelanodermia, espessamento e enrijecimento da pele de membros inferiores.

Foi feito uma análise dos exames de imagem apresentados pela paciente na consulta na UBS, em que apresentava uma tomografia computadorizada (TC) do dia 4 de agosto de 2018 que apresentou um padrão de vidro fosco bilateral compatível com diagnóstico de Pneumopatia Intersticial e bronquiectasias nas bases pulmonares. TC apresentada na figura 5 e laudo da TC no anexo 1. Ao esofagograma do dia 7 de agosto de 2018 apresentou dilatações esofagianas, preenchimento contínuo e fase tardia com retardo do esvaziamento por redução do calibre apresentados (anexo 2). 


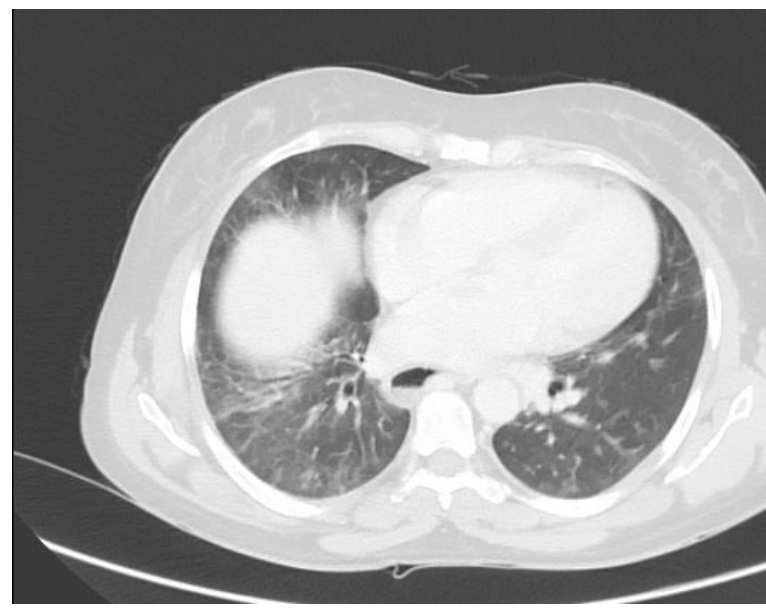

Figura 5. Pneumopatia intersticial. Paciente sexo feminino, 41 anos. Imagem coronal de TC de alta resolução no tórax. Com presença de opacidades em vidro fosco esparsas no parênquima pulmonar bilateralmente e bronquiectasias nas bases pulmonares

Foi referido no prontuário que após diagnóstico clínico de ES, a paciente foi encaminhada do ambulatório de dermatologia da Universidade de Gurupi para o HGP, onde foi internada e evoluiu com considerável melhora clínica da dor e edema dos membros superiores e inferiores, após o uso de analgesia e antibioticoterapia com Cefalotina 1 grama por 3 dias. No dia 7 de agosto no HGP, adotou-se uma intervenção terapêutica para PI através da prescrição da infusão da Ciclofosfamida 1 grama, sem intercorrências.

No dia 8 de agosto de 2018 a paciente teve alta hospitalar do HGP, sendo prescrito para uso em casa de Nifedipino Retard 20 mg de 8 em 8 horas, Captopril $25 \mathrm{mg}$ de 12 em 12 horas, Omeprazol $40 \mathrm{mg} 12$ em 12 horas, Domperidona $10 \mathrm{mg} 3$ vezes ao dia e Carbonato de Cálcio associado à Vitamina $\mathrm{D}$, sendo um comprimido após o almoço e o desmame da Prednisona.

Foi relatado no prontuário que a paciente no momento da alta hospitalar apresentava infecção na pele de dois pododáctilos do pé direito sendo também prescrito para uso em casa de Cefalexina $500 \mathrm{mg}$ de 6 em 6 horas por 5 dias. Foi solicitado também a coleta de hemograma entre os dias 17 a 21 de agosto de 2018 para avaliação da dose da próxima Ciclofasfamida que seria no dia 6 de setembro de 2018.

\section{DISCUSSÃO}

A Esclerodermia Sistêmica é cerca de quatro vezes mais frequente entre as mulheres, na faixa etária dos 35 aos 54 anos, faixa etária da paciente deste estudo (BRASIL, 2013).

A esclerodactilia, a esclerodermia e a leucomelanodermia apresentadas pela paciente são ttrês das manifestações clínicas clássicas da ES, resultando em comprometimento estrutural e funcional do organismo (SINGH et al., 2019).

A esclerodactilia está relacionada à disfunção vascular de pequenas veias e artérias e desenvolvimento de fibrose nos órgãos e tecidos afetados. O acúmulo de colágeno é relacionado a uma elevada quantidade de fibroblastos ativados que promovem uma fibrose cutânea excessiva ocasionando edema, endurecimento e atrofia articular (POPE, JOHNSON, 2015; SINGH et al., 2019).

A esclerodermia caracteriza-se pela perda das pregas cutâneas normais, da expressão facial e da capacidade de abrir completamente a boca, ou seja, a microstomia, associados de dentes a mostra, lábios adelgaçados e afinamento do nariz. Enquanto a leucomelanodermia resulta em hiperpigmentação da pele com despigmentação de aspecto salpicado em certas áreas (SINGH et al., 2019).

O FRy é desencadeado por alterações vasculares, onde a disfunção endotelial e o espessamento fibrótico da camada íntima formam a base da lesão microvascular esclerodérmica. O endotélio lesado produz maior quantidade de fatores vasoconstrictores e menor quantidade de substâncias vasodilatadoras resultando em vasoespasmo de pequenas artérias e arteríolas digitais e se apresenta na forma de palidez, cianose e rubor das regiões afetadas. 
Entretanto, por longos períodos, causa o desenvolvimento de pequenas úlceras dolorosas nas polpas digitais (POPE, JOHNSON, 2015; SINGH et al., 2019).

Outra consequência do processo fibrótico é a DRGE causada pela redução da pressão de repouso do esfíncter esofagiano inferior (FIKREE, 2019).

A PI está entre as principais causas de óbito. A mesma foi evidenciada pela TC que apresentou uma imagem de padrão de vidro fosco bilateral compatível com a afecção, que pode ser focal ou difuso, com presença de aspecto reticulado, em favo-de-mel ou de bronquiectasias (POPE, JOHNSON, 2015; FIKREE, 2019; SINGH et al., 2019).

A PI é causada pela ativação de células imunes e hiperplasia de fibroblastos, levando ao aumento da produção de colágeno, provocando fibrose pulmonar, reduzindo a capacidade pulmonar, o que justifica a dispneia aos mínimos esforços referida pela paciente (POPE, JOHNSON, 2015; SINGH et al., 2019).

Durante o tratamento, utilizou-se a Ciclofosfamida como terapia imunossupressora que é o padrão ouro para PI, contribuindo para o controle dos sintomas e melhor prognóstico da paciente. Quanto aos acometimentos vasculares foi prescrito $\mathrm{o}$ Nifedipino, droga de primeira escolha para controle dos sintomas agudos (BRASIL, 2013; SINGH et al., 2019).

Para prevenção de uma das mais frequentes complicações da doença, a crise renal esclerodérmica foi prescrito o Captopril e para prevenção de uma possível consequência de má absorção de nutrientes, que é a Osteoporose, foi prescrito o Carbonato de Cálcio com Vitamina D. Para diminuição dos sintomas gástricos foram prescritos o Omeprazol e a Domperidona (BRASIL, 2013; SINGH et al., 2019).
A Esclerodermia Sistêmica é uma patologia grave com intensas repercussões clínicas. O acometimento do trato gastrointestinal, articulações e pulmões são responsáveis um por grande impacto no prognóstico e qualidade de vida do paciente podendo repercutir em sua taxa de mortalidade. Dessa forma é essencial o diagnóstico precoce, pois permite o estabelecimento de intervenções terapêuticas específicas que podem ser determinantes no prognóstico do paciente.

Esse estudo presta contribuição à comunidade acadêmica e à sociedade, uma vez que são poucos os relatos de caso acerca dessa patologia, no entanto há necessidade de maiores estudos que a respeito de sua patogênese por se tratar de uma doença pouco conhecida e de difícil diagnóstico.

\section{REFERÊNCIAS}

BRASIL. Ministério da Saúde. Esclerose sistêmica. Portaria SAS/MS n. 99, de 7 de fevereiro de 2013. Lex: Protocolo Clínico e Diretrizes Terapêuticas, Brasília, p. 289-310, fev., 7. 2013. Protocolo Clínico e Diretrizes Terapêuticas.

Dermatologia / Rubem David Azulay, David Rubem Azulay, Luna Azulay-Abulafia. 7. ed. Rio de Janeiro: Guanabara Koogan, 2017.

FIKREE, Asma. Scleroderma and other connective tissue disorders. Medicine, [s.l.], v. 47, n. 7, p.460465, jul. 2019. Elsevier BV. http://dx.doi.org/10.1016/j.mpmed.2019.04.012.

JACQUIER, Marine et al. Scleroderma Renal Crisis in a Systemic Sclerosis With Anti-PM/Scl Antibodies. Kidney International Reports, [s.1.], p.1-4, jul. 2019. Elsevier $\mathrm{BV}$. http://dx.doi.org/10.1016/j.ekir.2019.07.002.

POPE, Janet E.; JOHNSON, Sindhu R.. New Classification Criteria for Systemic Sclerosis (Scleroderma). Rheumatic Disease Clinics Of North America, [s.1.], v. 41, n. 3, p.383-398, ago. 2015. Elsevier BV. http://dx.doi.org/10.1016/j.rdc.2015.04.003. 
SINGH, Deependra et al. Scleroderma: An insight into causes, pathogenesis and treatment strategies. Pathophysiology, [s.l.], v. 26, n. 2, p.103-114, jun. 2019. Elsevier $\mathrm{BV}$. http://dx.doi.org/10.1016/j.pathophys.2019.05.003.

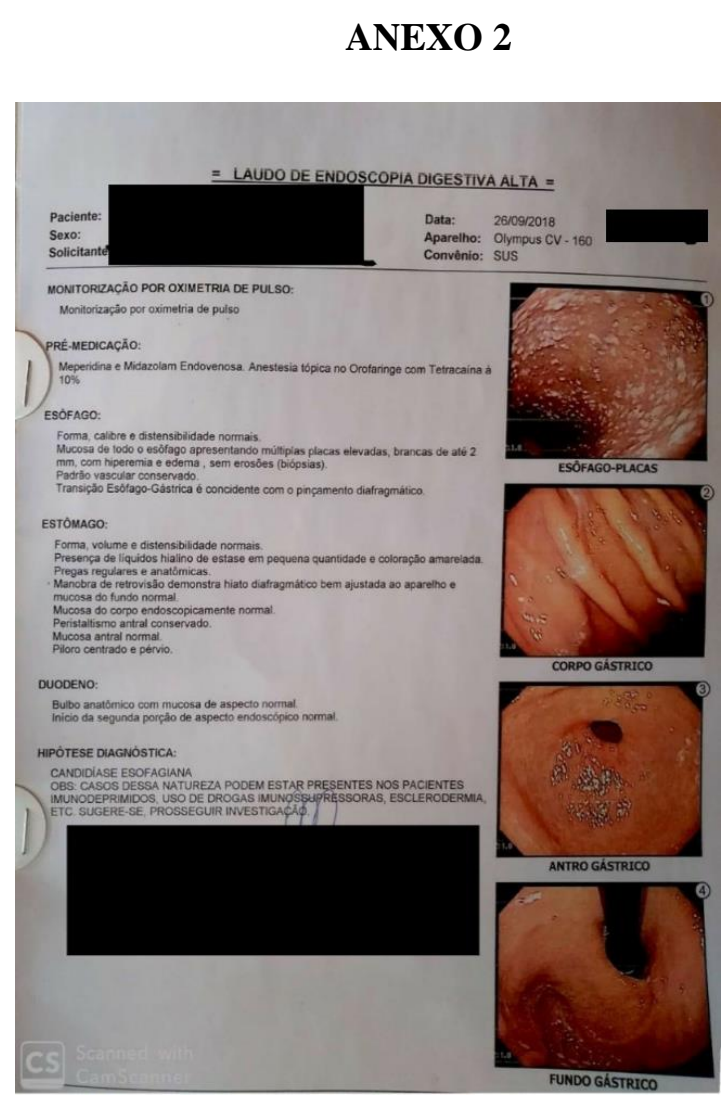


ANEXO 3

\section{RELATÓRIO DE PATOLOGIA CIRÚRGICA}

\begin{tabular}{|c|c|}
\hline $\begin{array}{l}\text { Informaçóes clinicas } \\
\text { disponibillzadas }\end{array}$ & Hipotese clinica de monillase. \\
\hline \multicolumn{2}{|l|}{$\begin{array}{l}\text { Nieroscopia e parecer } \\
\text { diagnóstico }\end{array}$} \\
\hline & Esodtago \\
\hline & ESOFAGITE CRONICA LEVE \\
\hline & $\begin{array}{l}\text { - A pesquisa histoquimica para fungos resultou positiva para raras estruturas compativeis } \\
\text { com pseudo-hilas de Candida spp. }\end{array}$ \\
\hline
\end{tabular}

$\overline{\text { Macroscopla }}$

\section{Esòfago}

Número de fragmentos: 03

Medidas: 0,3 a $0,4 \mathrm{~cm}$

Cassete 1 - 1800355544 Todo material è submetido a exame histológico
B. 1
L. 3
C. Grocoth |HE IPAS 